\title{
Análise histopatológica da cérvice uterina de mulheres em cárcere de Mato Grosso
}

\author{
Histopathological analysis of the uterine cervix of women in \\ prison in Mato Grosso
}

\author{
1 Aline da Silva Caldeira aline_silvacaldeira@hotmail.com \\ 2 Ana Cláudia Pereira Terças-Trettel \\ 3 Vagner Ferreira do Nascimento \\ 4 Thalise Yuri Hattori \\ 5 Andreza Lucia Menezes
}

\footnotetext{
1 Egressa do Departamento de Enfermagem, Universidade do Estado de Mato Grosso - UNEMAT, Tangará da Serra.

2 Doutora em Medicina Tropical. Departamento de Enfermagem, Universidade do Estado de Mato Grosso - UNEMAT, Tangará da Serra. Programa de Pós graduação em Saúde Coletiva da Universidade Federal de Mato Grosso - UFMT.

3 Doutor em Bioética. Departamento de Enfermagem, Universidade do Estado de Mato Grosso - UNEMAT, Tangará da Serra.

4 Mestre em Ciências da Saúde. Departamento de Enfermagem, Universidade do Estado de Mato Grosso - UNEMAT, Tangará da Serra.

5 Mestre em Citologia. Instituto Arel luga, Cuiabá -MT.
}

\section{RESUMO}

A neoplasia de colo de útero impacta na morbimortalidade de mulheres no mundo e, quando diagnosticado precocemente, as chances de cura são significantes. Assim, a utilização do teste de Papanicolau é uma ferramenta primordial. Nesta pesquisa, objetivou-se analisar achados histopatológicos da cérvice uterina de mulheres em cárcere de Mato Grosso. Trata-se de estudo de corte aberta, em que a coleta de dados foi realizada sequencialmente em 2017 e 2018, por meio de entrevista semiestruturada e coleta de amostra da cérvice uterina pela técnica de Papanicolau. A amostra do estudo foi composta predominantemente de mulheres jovens, de cor parda, renda mensal de um a dois salários mínimos, detidas por tráfico, e que já haviam realizado exame preventivo em algum momento de sua vida. As alterações benignas ou reparativas foram observadas em ambos os anos da análise. Os achados deste estudo mostraram resultados satisfatórios com relação à análise histopatológica das mulheres, indicando a presença de saúde.

\section{Palavras-chave:}

Enfermagem no consultório. Ginecologia. Prisões. Saúde da mulher. Teste Papanicolau.

\begin{abstract}
Cervical neoplasia impacts the morbidity and mortality of women worldwide, when diagnosed early, the chances of a cure are significant, so the use of the Pap test is a primary tool. This research aims to analyze histopathological findings of the uterine cervix of women in prison in Mato Grosso. This is an open cohort study, in which data collection was performed sequentially in 2017 and 2018, through semi-structured interviews and sampling of the uterine cervix by the Pap smear. The study sample was composed predominantly of young women, brown in color, monthly income of one to two minimum wages, detained for trafficking, and who had already undergone preventive examination at some point in their life. Benign or reparative changes were observed in both years of the analysis. The findings of this study showed satisfactory results regarding the histopathological analysis of the women, indicating the presence of health.
\end{abstract}

\section{Keywords:}

Office Nursing. Gynecology. Prisons. Women's health. Papanicolau test.

\section{Como você deve citar?}

CALDEIRA, Aline da Silva et al. Análise histopatológica da cérvice uterina de mulheres em cárcere de Mato Grosso. Cadernos UniFOA, Volta Redonda, n. 45, p. 121-132, abril 2021. 


\section{INTRODUÇÃO}

O câncer de colo de útero (CCU) é considerado um importante problema de saúde pública, sendo uma das principais causas de morbimortalidade feminina e com progressivo impacto na qualidade de vida dessa população, principalmente nos países em desenvolvimento (SIMÕES et al., 2014). Está entre os sete principais tipos de cânceres mais prevalentes, sendo o terceiro mais comum em mulheres e a quarta causa de morte no Brasil (OMS, 2019a).

O Instituto Nacional de Câncer (Inca) estimou, para o Brasil, 16.370 novos casos para 2019, com prevalência de 15,43 casos a cada 100 mil mulheres. No entanto, até o mês de maio de 2019, foram registrados no mundo 569.847 novos casos e 311.365 mortes (OMS, 2019b). Na região Centrooeste e no estado é o terceiro câncer mais comum entre as mulheres com 1.490 casos e 340 casos, respectivamente, em 2018 (BRASIL, 2017). Dessa forma, o diagnóstico tardio traz um desfecho clínico desfavorável. Nesse contexto, o exame preventivo é a principal estratégia para identificar alterações na cérvice, atuando como importante ferramenta no cuidado e prevenção da doença (AMARAL et al., 2017; OMS, 2014; SILVEIRA et al., 2018). Conforme protocolo estabelecido pelo Ministério da Saúde (MS), para rastreamento, o exame deve ser realizado a partir dos 25 anos e sequencialmente, com periodicidade anual (BRASIL, 2016). Essa conduta preventiva poderá identificar precocemente as lesões precursoras do CCU, que são denominadas neoplasias intraepiteliais cervicais (NIC) de grau II ou III, as quais possuem evolução lenta e elevado índice de cura.

Todavia, o cenário brasileiro retrata uma dificuldade de adesão ao exame preventivo, já que $40 \%$ das mulheres nunca realizaram o exame, apesar da oferta e gratuidade por meio do Sistema único de Saúde (SUS). Um dos motivos para não realização do preventivo pode estar relacionado às desigualdades no acesso aos serviços de saúde no país (CERQUEIRA et al., 2017), especialmente para mulheres em situação de prisão (TERÇAS et al., 2019), que, além de não serem assistidas em suas demandas em saúde, estão expostas a fatores de risco adicionais como celas superlotadas, insalubridade, violência, promiscuidade e doenças infectocontagiosas, sendo a última um dos principais motivos causadores do CCU (FRANCISCO, 2015; ALMEIDA et al., 2015; CARVALHO et al., 2016; MAGALHÃES et al., 2017; GRAÇA et al., 2018a).

É importante ressaltar que mulheres privadas de liberdade possuem históricos de saúde que normalmente determinam os comportamentos de risco e redução de autocuidado, além ser importante observar que estão mais vulneráveis ao adoecimento e prejuízos quanto à saúde ginecológica frente a outras mulheres. E como raramente há controle e acompanhamento da saúde desse grupo durante o período de reclusão, deixam de realizar o exame preventivo e outros cuidados importantes para prevenção do $\mathrm{CCU}$, que pode ocasionar o adoecimento lento e silencioso. Assim, o estudo objetivou analisar os achados histopatológicos da cérvice uterina de reeducandas.

\section{METODOLOGIA}

Trata-se de estudo de coorte aberto, realizado em dois anos sequenciais, de 2017 e 2018, que foi desenvolvido em uma cadeia pública feminina na região médio norte de Mato Grosso, inaugurada em 1978. Sua capacidade máxima, em 2019, era para 60 reeducandas em oito celas, atendendo vários municípios de Mato Grosso e outros estados próximos (GRAÇA et al., 2018b).

Foram incluídas no estudo mulheres condenadas ou em regime provisório, e que no momento, corresponderam ao quantitativo geral de reeducandas na instituição. Ao final, participaram 66 mulheres, sendo 42, no ano de 2017, e 35, em 2018. Dessas mulheres, 11 estiveram presentes em ambos os anos 
da coorte (Figura 1). Ressalta-se que todas aceitaram participar da pesquisa, assinando um Termo de Consentimento Livre e Esclarecido (TCLE). As mulheres que receberam habeas corpus até a data da coleta de dados foram excluídas.

Figura 1 - Constituição das coortes de 2017 e 2018. Cadeia feminina, Mato Grosso, 2019.

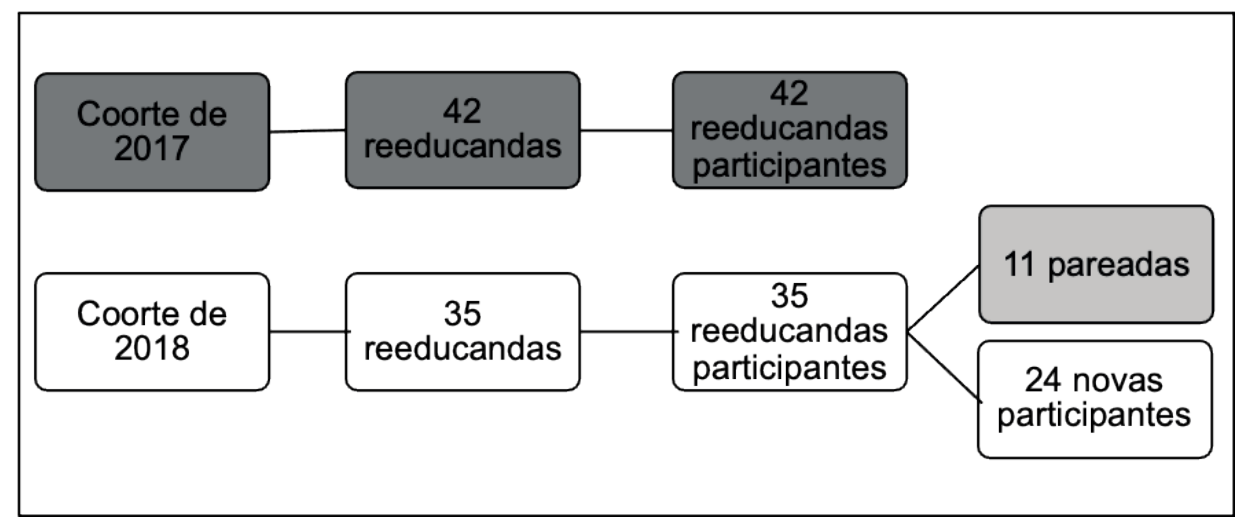

Fonte: Autores

As coletas de dados foram realizadas em duas etapas. A primeira se deu por meio de entrevista realizada em ambiente reservado, com duração de trinta minutos para cada reeducanda com utilização de formulário semiestruturado, contendo perguntas abertas e fechadas que abordavam as variáveis relacionadas ao perfil socioeconômico (idade, cor, escolaridade), antecedentes ginecológicos (motivo da realização do $\mathrm{CCO}$, realização anterior de $\mathrm{CCO}$, ano do último exame, utilização de DIU, estar gestante, uso de anticoncepcional oral (ACO), utilização de reposição hormonal, radioterapia pregressa, histórico de sangramento após ato sexual, data da última menstruação e sangramento após início de menopausa).

$\mathrm{Na}$ segunda etapa, foi realizada consulta de enfermagem seguindo os protocolos do Ministério da Saúde e as etapas da Sistematização da Assistência de Enfermagem (SAE) para assistência ginecológica. A realização do exame colpocitológico ocorreu durante o exame ginecológico e a amostra com o material coletado foi fixado em lâmina com solução de propilenoglicol e álcool absoluto, sendo posteriormente encaminhada para laboratório.

Os resultados da análise laboratorial compuseram as variáveis dos achados histopatológicos (epitélios apresentados na amostra, condição do epitélio dento do padrão de normalidade ou presença de células atípicas de significado intermediário, presença de células atípicas em células escamosas, presença de células atípicas em células glandulares, outras neoplasias, presença de células endometriais e outras observações).

A organização dos dados ocorreu por meio da sistematização em planilhas eletrônicas pelo método de dupla digitação. Os dados foram confrontados e conferidos pelo programa Data Compareò. Após a completitude dos dados e construção do banco, houve a importação para o Statistical Package for the Social Sciences (SPSS) versão 20.0, no qual realizou-se a análise descritiva dos dados (frequência absoluta e relativa), sendo os resultados apresentados por meio de tabelas. A análise laboratorial das lâminas ocorreu no laboratório do Instituto Arel Liga, em Cuiabá, e os laudos foram emitidos por profissional citopatologista credenciado ao SUS.

A pesquisa respeitou os aspectos éticos de pesquisa com seres humanos, de acordo com a Resolução 466/12 e foi aprovada pelo Comitê de Ética em Pesquisa (CEP) com seres humanos da Universidade do Estado de Mato Grosso, sob CAAE 50417815.8.0000.5166 e parecer 1.457.621/2016. 
A presente pesquisa recebe apoio financeiro da Fundação de Amparo à Pesquisa do Estado de Mato Grosso (Fapemat), por meio do Edital PPSUS 003/2017, processo numérico 285300/2018.

\section{RESULTADOS}

0 perfil das reeducandas é caracterizado em sua maioria por mulheres com idade entre 30 a 39 anos, em 2017, e no ano subsequente, mulheres mais jovens (18 a 29 anos). Em ambos os anos, a maior frequência foi de mulheres de cor parda (69,04\% e 74,29\%), com ensino fundamental $(50,00 \%$ e $54,29 \%$ ), de renda familiar entre de 1 e 2 salários mínimos (52,38\% e 60,00\%), sendo o tráfico de drogas o principal motivo de detenção $(64,29 \%$ e $60,00 \%$ ) (Tabela 1). Analisando as reeducandas presentes no biênio (11 pareadas), o perfil foi similar ao descrito em relação ao total de mulheres do estudo, ou seja, predominando mulheres dos 30 aos 39 anos $(45,45 \%)$, pardas $(90,91 \%)$, com ensino fundamental $(54,55 \%)$, renda de um a dois salários mínimos (54,55\%) e todas detidas por tráfico de drogas.

Tabela 1 - Aspectos sociodemográficos de reeducandas de uma cadeia pública feminina de Mato Grosso nos anos de $2017(\mathrm{~N}=42), 2018(\mathrm{~N}=35)$ e ambos anos ( $\mathrm{N}=11$ pareadas). 2019. Tangará da Serra - MT, Brasil.

\begin{tabular}{|c|c|c|c|c|c|c|c|}
\hline \multicolumn{2}{|c|}{ Variáveis } & \multicolumn{2}{|c|}{$2017(\mathrm{~N}=42)$} & \multicolumn{2}{|c|}{$2018(\mathrm{~N}=35)$} & \multicolumn{2}{|c|}{ Pareadas $(\mathrm{N}=11)$} \\
\hline & & $\mathbf{N}$ & $\%$ & $\mathbf{N}$ & $\%$ & $\mathbf{N}$ & $\%$ \\
\hline \multirow{4}{*}{ Faixa etária } & $18-29$ anos & 15 & 35,71 & 19 & 54,29 & 04 & 36,36 \\
\hline & $30-39$ anos & 19 & 45,24 & 09 & 25,71 & 05 & 45,45 \\
\hline & $40-49$ anos & 06 & 14,29 & 07 & 20,00 & 02 & 18,19 \\
\hline & $50-60$ anos & 02 & 4,76 & 00 & - & 00 & - \\
\hline \multirow{4}{*}{ Cor } & Branca & 05 & 11,90 & 06 & 17,14 & 01 & 9,09 \\
\hline & Negra & 07 & 16,67 & 03 & 8,57 & 00 & - \\
\hline & Parda & 29 & 69,04 & 26 & 74,29 & 10 & 90,91 \\
\hline & Amarela & 01 & 2,38 & 00 & - & 00 & - \\
\hline \multirow{4}{*}{ Escolaridade } & Sem Escolaridade & 01 & 2,38 & 00 & - & 01 & 9,09 \\
\hline & Ensino Fundamental & 21 & 50,00 & 19 & 54,29 & 06 & 54,55 \\
\hline & Ensino Médio & 18 & 42,86 & 16 & 45,71 & 04 & 36,36 \\
\hline & Ensino Superior & 02 & 4,76 & 00 & - & 00 & - \\
\hline \multirow{4}{*}{ Renda Familiar } & 1 a 2 salários mín.* & 22 & 52,38 & 21 & 60,00 & 06 & 54,55 \\
\hline & 3 a 4 salários mín. & 10 & 23,81 & 04 & 11,43 & 01 & 9,09 \\
\hline & 5 a 6 salários mín. & 02 & 4,76 & 01 & 2,86 & 01 & 9,09 \\
\hline & Não tinha renda & 08 & 19,05 & 09 & 25,71 & 03 & 27,27 \\
\hline \multirow{5}{*}{$\begin{array}{l}\text { Motivo da } \\
\text { Detenção }\end{array}$} & Tráfico & 27 & 64,29 & 21 & 60,00 & 11 & 100,00 \\
\hline & Homicídio & 09 & 21,43 & 08 & 22,86 & - & - \\
\hline & Roubo & 01 & 2,38 & 04 & 11,43 & - & - \\
\hline & Furto & 01 & 2,38 & 00 & - & - & - \\
\hline & Outros** & 04 & 9,52 & 02 & 5,71 & - & - \\
\hline
\end{tabular}

*o salário mínimo em 2017 correspondeu a R\$ 937,00 e em 2018 R\$ 954,00.

** outros crimes foram: furto, organização de quadrilha, latrocínio, roubo, danos ao patrimônio público e porte de armas.

Fonte: Autores

Os antecedentes ginecológicos dessas mulheres foram expressos na tabela 2, em que se observa que grande parte já havia realizado o exame alguma vez, sem utilização de dispositivo intrauterino (DIU), ACO e hormônio para menopausa ou radioterapia. Seis (14,29\%) relataram sangramento após as 
relações sexuais, uma $(2,38 \%)$ informou ter apresentado sangramento após a menopausa e apenas uma estava gestante em 2017. Os sinais de infecções sexualmente transmissíveis (IST) foram identificados em $16(38,10 \%)$ mulheres, em 2017, e no ano seguinte, em apenas três (8,57\%).

Sob a ótica das mulheres atendidas em ambos os anos ( $\mathrm{N}=11$ pareadas) predominou aquelas com exame realizado em algum momento de sua vida e sem DIU. Em 2018, 27,27\% passaram a utilizar anticoncepcional e relataram com unanimidade não estarem na menopausa. Não houve relatos de sangramento após as relações sexuais, porém cinco mulheres $(45,45 \%)$ apresentaram sinais de IST no primeiro ano e nenhuma no segundo ano (Tabela 2). 
Tabela 2 - Antecedentes ginecológico de reeducandas de uma cadeia pública feminina de Mato Grosso nos anos de 2017 ( $N=42), 2018$ ( $N=35)$, e presentes nos 2 anos ( $N=11$ pareadas). 2019. Tangará da Serra - MT, Brasil.

\begin{tabular}{|c|c|c|c|c|c|c|c|c|c|}
\hline \multirow{3}{*}{ Variáveis } & \multicolumn{5}{|c|}{$2017(\mathrm{~N}=42)$} & \multicolumn{4}{|c|}{$2018(\mathrm{~N}=35)$} \\
\hline & \multicolumn{3}{|c|}{ Total da amostra( $\mathrm{N}=42)$} & \multicolumn{2}{|c|}{$\begin{array}{c}\text { Pareadas } \\
(\mathrm{N}=11)\end{array}$} & \multicolumn{2}{|c|}{$\begin{array}{c}\text { Total da } \\
\text { amostra(N=35) }\end{array}$} & \multicolumn{2}{|c|}{$\begin{array}{c}\text { Pareadas } \\
(\mathrm{N}=11)\end{array}$} \\
\hline & $\mathbf{N}$ & & $\%$ & $\mathbf{N}$ & $\%$ & $\mathbf{N}$ & $\%$ & $\mathbf{N}$ & $\%$ \\
\hline \multirow{2}{*}{ Fez exame alguma vez } & Sim & 37 & 88,10 & 09 & 81,82 & 28 & 80,00 & 11 & 100,00 \\
\hline & Não & 05 & 11,90 & 02 & 18,18 & 07 & 20,00 & 00 & - \\
\hline \multirow{3}{*}{ Usa DIU } & Sim & 00 & - & 00 & - & 00 & - & 00 & - \\
\hline & Não & 39 & 92,86 & 09 & 81,82 & 35 & 100,00 & 11 & 100,00 \\
\hline & Não sabe & 03 & 7,14 & 02 & 18,18 & 00 & - & 00 & - \\
\hline \multirow{3}{*}{ Está Gravida } & Sim & 01 & 2,38 & 01 & 9,09 & 00 & - & 00 & - \\
\hline & Não & 37 & 88,10 & 08 & 72,73 & 35 & 100,00 & 11 & 100,00 \\
\hline & Não sabe & 04 & 9,52 & 02 & 18,18 & 00 & - & 00 & - \\
\hline \multirow{3}{*}{ Usa ACO } & Sim & 05 & 11,90 & 00 & - & 10 & 28,57 & 03 & 27,27 \\
\hline & Não & 34 & 80,95 & 09 & 81,82 & 25 & 71,43 & 08 & 72,73 \\
\hline & Não sabe & 03 & 7,14 & 02 & 18,18 & 00 & - & 00 & - \\
\hline \multirow{3}{*}{$\begin{array}{l}\text { Usa hormônio } \\
\text { menopausa }\end{array}$} & Sim & 00 & - & 00 & - & 00 & - & 00 & - \\
\hline & Não & 39 & 92,86 & 11 & 100,00 & 35 & 100,00 & 11 & 100,00 \\
\hline & Não sabe & 03 & 7,14 & 00 & - & 00 & - & 00 & - \\
\hline \multirow{2}{*}{$\begin{array}{l}\text { Sangramento após } \\
\text { relação sexual }\end{array}$} & Sim & 01 & 2,38 & 01 & 9,09 & 00 & - & 00 & - \\
\hline & Não & 41 & 97,62 & 10 & 90,91 & 35 & 100,00 & 11 & 100,00 \\
\hline \multirow{2}{*}{$\begin{array}{l}\text { Sangramento após } \\
\text { menopausa }\end{array}$} & Sim & 01 & 2,38 & 01 & 9,09 & 00 & - & 00 & - \\
\hline & Não & 41 & 97,62 & 10 & 90,91 & 35 & 100,00 & 11 & 100,00 \\
\hline \multirow{2}{*}{ Sinais de IST } & Sim & 16 & 38,10 & 05 & 45,45 & 03 & 8,57 & 00 & - \\
\hline & Não & 26 & 61,90 & 06 & 54,55 & 32 & 91,43 & 11 & 100,00 \\
\hline
\end{tabular}

Fonte: Autores

Os achados dos exames colpocitológicos do total das reeducandas apontaram para presença em ambos os anos de epitélios escamoso em todas as amostras; já o epitélio glandular foi encontrado em 
34 reeducandas (80,95\%), no ano de início, e 16 (45,29\%), em 2018. No entanto, epitélios metaplásicos foram evidenciados apenas em cinco mulheres (11,90\%), em 2017, e oito $(22,86 \%)$, no ano seguinte. As características histológicas visualizadas apontaram que a maioria das amostras da cérvice uterina não estava dentro do padrão de normalidade em ambos os anos, sendo que as principais alterações encontradas foram alterações celulares benignas ou reparativas (85,71\%, em 2017, e 88,57\%, em 2018), porém duas $(4,76 \%)$ apresentaram atipias celulares de significado indeterminado em ambos os anos e duas $(5,71 \%)$, atipias em células escamosas, no segundo ano (Tabela 3$)$.

Tabela 3 - Achados no exame colpocitológico de reeducandas de uma cadeia pública feminina de Mato Grosso nos anos de 2017 ( $\mathrm{N}=42), 2018(\mathrm{~N}=35)$, e presentes nos 2 anos ( $\mathrm{N}=11$ pareadas). 2019.

Tangará da Serra - MT, Brasil.

\begin{tabular}{|c|c|c|c|c|c|c|c|c|c|c|}
\hline \multicolumn{3}{|c|}{ Variáveis } & \multicolumn{4}{|c|}{$2017(\mathrm{~N}=42)$} & \multicolumn{4}{|c|}{$2018(\mathrm{~N}=35)$} \\
\hline & & & \multicolumn{2}{|c|}{$\begin{array}{c}\text { Total da } \\
\text { amostra( }(\mathrm{N}=42)\end{array}$} & \multicolumn{2}{|c|}{$\begin{array}{l}\text { Pareadas } \\
(\mathrm{N}=11)\end{array}$} & \multicolumn{2}{|c|}{$\begin{array}{c}\text { Total da } \\
\text { amostra(N=35) }\end{array}$} & \multicolumn{2}{|c|}{$\begin{array}{l}\text { Pareadas } \\
(\mathrm{N}=11)\end{array}$} \\
\hline & & & $\mathbf{N}$ & $\%$ & $\mathbf{N}$ & $\%$ & $\mathbf{N}$ & $\%$ & $\mathbf{N}$ & $\%$ \\
\hline \multirow{6}{*}{ Epitélios } & \multirow{2}{*}{ Escamoso } & Sim & 42 & 100,00 & 11 & 100,00 & 35 & 100,00 & 11 & 100,00 \\
\hline & & Não & 00 & - & 00 & - & 00 & - & 00 & - \\
\hline & \multirow{2}{*}{ Glandular } & Sim & 34 & 80,95 & 09 & 81,82 & 16 & 45,71 & 05 & 45,45 \\
\hline & & Não & 08 & 19,05 & 02 & 18,18 & 19 & 54,29 & 06 & 54,55 \\
\hline & \multirow{2}{*}{ Metaplásico } & Sim & 05 & 11,90 & 02 & 18,18 & 08 & 22,86 & 08 & 72,73 \\
\hline & & Não & 37 & 88,10 & 09 & 81,82 & 27 & 77,14 & 03 & 27,27 \\
\hline \multirow{2}{*}{\multicolumn{2}{|c|}{$\begin{array}{l}\text { Dentro dos padrões de } \\
\text { normalidade }\end{array}$}} & Sim & 03 & 7,14 & 01 & 9,09 & 04 & 11,43 & 03 & 27,27 \\
\hline & & Não & 39 & 92,86 & 10 & 90,91 & 31 & 88,57 & 08 & 72,73 \\
\hline \multirow{2}{*}{\multicolumn{2}{|c|}{$\begin{array}{l}\text { Alterações celulares } \\
\text { benignas ou reparativas }\end{array}$}} & Sim & 36 & 85,71 & 10 & 90,91 & 31 & 88,57 & 08 & 72,73 \\
\hline & & Não & 06 & 14,29 & 01 & 9,09 & 04 & 11,43 & 03 & 27,27 \\
\hline \multirow{6}{*}{$\begin{array}{l}\text { Células } \\
\text { atípicas }\end{array}$} & \multirow{2}{*}{$\begin{array}{l}\text { Significado } \\
\text { indeterminado }\end{array}$} & Sim & 02 & 4,76 & 00 & - & 02 & 5,71 & 00 & - \\
\hline & & Não & 40 & 95,24 & 11 & 100,00 & 33 & 94,29 & 11 & 100,00 \\
\hline & \multirow{2}{*}{ Escamosas } & Sim & 00 & - & 00 & - & 02 & 5,71 & 00 & - \\
\hline & & Não & 42 & 100,00 & 11 & 100,00 & 33 & 94,29 & 11 & 100,00 \\
\hline & \multirow{2}{*}{ Metaplásico } & $\operatorname{Sim}$ & 05 & 11,90 & 02 & 18,18 & 08 & 22,86 & 08 & 72,73 \\
\hline & & Não & 37 & 88,10 & 09 & 81,82 & 27 & 77,14 & 03 & 27,27 \\
\hline \multirow{2}{*}{\multicolumn{2}{|c|}{ Outras neoplasias malignas }} & Sim & 00 & - & 00 & - & 00 & - & 00 & - \\
\hline & & Não & 42 & 100,00 & 11 & 100,00 & 35 & 100,00 & 11 & 100,00 \\
\hline \multirow{2}{*}{\multicolumn{2}{|c|}{$\begin{array}{l}\text { Presença de células } \\
\text { endometriais }\end{array}$}} & Sim & 00 & - & 00 & - & 00 & - & 00 & - \\
\hline & & Não & 42 & 100,00 & 11 & 100,00 & 35 & 100,00 & 11 & 100,00 \\
\hline
\end{tabular}

Fonte: Autores

Analisando os resultados das amostras pareadas coletadas, obteve-se, no biênio, presença de epitélio escamoso em sua totalidade. A presença do epitélio glandular apresentou redução de $81,82 \%$, no primeiro ano, para $45,45 \%$, no segundo. A situação inversa foi observada com epitélio metaplásico, com aumento de 18,18\%, em 2017, para 72,73\%, em 2018.

As 11 reeducandas acompanhadas de 2017 a 2018 apresentaram redução nas alterações no padrão de normalidade e alterações benignas reparativas das amostras da cérvice uterina de $90,91 \%$, 
em 2017 , para $72,73 \%$, em 2018. Nenhuma das amostras apresentaram atipias de significado indeterminado, escamoso, glandular, outras neoplasias ou presença de células endometriais no biênio analisado.

\section{DISCUSSÃO}

O perfil sociodemográfico das participantes se assemelha com estudos de outras regiões do país e do mundo com grande número de mulheres jovens, pardas e com baixa escolaridade (BRONSON e CARSON, 2019; EVARISTO et al., 2017; ICPR, 2019). Outro estudo demonstra similaridades em relação à renda familiar baixa e ao motivo da detenção ser o tráfico de drogas (GERMANO et al., 2018).

Os aspectos que envolvem os antecedentes ginecológicos dessas mulheres são semelhantes aos descritos em estudos nacionais, uma vez que as mulheres que se submetem ao exame ginecológico já o realizaram anteriormente em algum período de sua vida (GRAÇA et al., 2018b; BORGES et al., 2018; TERÇAS et al., 2019). Entretanto, uma parcela significativa dessas mulheres relatou que não obteve acesso ao atendimento ginecológico e exame para prevenção do CCU, mesmo possuindo vários anos de experiência sexual.

Dentre os métodos contraceptivos utilizados pelas mulheres em situação de prisão, houve predomínio do uso de contraceptivos orais e ausência de outros métodos, como o DIU, por exemplo, similar ao descrito em pesquisa nacional (BISPO et al., 2016). Tais resultados podem ser justificados pela restrição ao acesso dos contraceptivos, pois não há o fornecimento pelo serviço de saúde prisional e, além disso, na maioria das cadeias, não há equipe de saúde, ficando sob responsabilidade dos familiares a sua aquisição, situação agravada pelo abandono familiar vivenciado por essas mulheres (TERÇAS et al., 2019).

Estudo conduzido em Mato Grosso (BORGES et al., 2018) apontou que as queixas ginecológicas, em 2016, eram pouco frequentes em reeducandas, assim como em pesquisa baiana (OLIVEIRA e SANTOAS, 2016), que identificou baixa frequência de queixas em relação às IST nessa população no nordeste brasileiro. No entanto, na observação clínica, foi expressivo os sinais de IST no estudo de coorte 2017; já no ano seguinte, houve uma redução, podendo estar relacionada às ações educativas que foram desenvolvidas pela equipe de pesquisa e acesso à consulta de enfermagem (CORSINO et al., 2018).

Outras queixas ginecológicas foram pouco frequentes, como, por exemplo, o sangramento após o ato sexual, que, no ambiente prisional, pode não ser uma evidência importante para direcionar suspeitas clínicas, pois muitas mulheres praticam o homossexualismo transitório na prisão (TERÇAS et al., 2019), não havendo penetração, o que reduz o atrito no canal vaginal e consequente presença de sangramento.

Os achados laboratoriais do biênio apontaram para a existência uniforme de células escamosas, presença evidente do epitélio glandular das amostras, no primeiro ano, e redução considerável, no ano seguinte. Já a presença de células metaplásicas foi pouco incidente, porém, relevante, com maior frequência no segundo ano analisado, indicando que as mucosas uterinas dessas mulheres apresentaram lesões possivelmente por agentes causadores de inflamação, realidade similar às pesquisas desenvolvidas no sul do país (BERTONCIM et al., 2019).

As alterações benignas ou reparativas estiveram presente no estudo em grande parte das amostras, no ano de 2017 , sinalizando a presença de alterações reativas nas células, porém sem malignidade considerável. No entanto, esses achados podem ser subjetivos para direcionar um desfecho definitivo (ROSENDO et al., 2019).

A observação e monitoramento das mudanças desses epitélios, no biênio, foi uma importante ferramenta preventiva, pois possibilitou verificar a melhoria de achados que expressa a saúde gineco- 
lógica como o aumento na identificação no epitélio metaplásico, passando de dois, no primeiro ano, para oito, no segundo, e diminuição nas alterações celulares benignas ou reparativas de dez para oito (SILVEIRA et al., 2018; OMS, 2019a).

Outras alterações epiteliais ou lesões com malignidades importantes não foram detectadas. É importante destacar que a incidência de lesões intraepiteliais de alto grau apresenta baixa ocorrência e se distribui de forma variável na população geral brasileira, como nas análises realizadas em Goiás, com 0,17\% de incidência; em São Paulo, de 2\%; Acre, com 2\%; Bahia, 0,3\%; e Rio Grande do Sul, 0,17\% (PINTO et al., 2011).

A detecção de achados benignos, neste estudo, remete à importância de divulgação para a comunidade científica, pois essa observação poderá direcionar novas pesquisas, conduzindo para ampliação de discussões sobre o tema e para o reconhecimento dos benefícios de saúde no ambiente carcerário, pois a promoção e a prevenção de agravos são extremamente relevantes nessa população, além da economia de tempo e recursos (GUIMARÃES et al., 2018). Ademais, é importante refletir que, mesmo que a unidade prisional não possua o número mínimo de recuperandas para a implementação de uma equipe de saúde, estratégias preventivas devem ser criadas, sejam através de redes entre serviços de saúde, como também, quando possível, com as instituições de ensino.

Assim, em se tratando dessa população em que o acesso a esse exame é limitado e pontual, é imprescindível que todas as etapas da prevenção sejam realizadas com excelência, pois poderão influenciar diretamente no diagnóstico e manejo clínico precoce (CORRÊA e FRANCISCO, 2018).

\section{CONCLUSÃO}

Observou-se, nos dois anos de análise, a presença de alterações celulares benignas ou reparativas, com apenas $4,76 \%$ de atipias celulares de significado indeterminado em ambos os anos e $5,71 \%$ atipias em células escamosas, no segundo ano da análise. 0 acompanhamento por dois anos consecutivos das características histopatológicas da cérvice uterina dessas mulheres pode reforçar a importância da realização anual do Papanicolau como principal ferramenta na prevenção do CCU.

A limitação do estudo está relacionada ao tamanho reduzido da amostra pareada, revelando achados que divergem da realidade de outras regiões, sendo essencial que sejam desenvolvidas novas pesquisas direcionadas às condições ginecológicas desse segmento de população, a fim de contribuir com a redução dos indicadores de morbimortalidade por esse agravo.

\section{REFERÊNCIAS}

ALMEIDA, P.R.C.; SOARES, R.S.C.; COURA, A.S.; CAVALCANTI, A.L.; DUTRA, M.O.M.; LIMA, T.M.A. Condição de saúde de mulheres privadas de liberdade: uma revisão integrativa. Rev.Bras. Ciên. da Saúde. n. 19, v. 1, p. 73-80, 2015. Disponível em: http://www.periodicos.ufpb.br/ojs/index.php/rbcs/article/view/23890. Acesso em: 10 dez. 2019

AMARAL, M.S.; GONÇALVES, A.G.; SILVEIRA, L.C.M. Prevenção do câncer de colo de útero: a atuação do profissional enfermeiro nas unidades básicas de saúde. Rev. Cientifica FacMais. n. 7, v. 1, p. 197223, 2017. Disponível em: http://revistacientifica.facmais.com.br/wp-content/uploads/2017/04/8PREVEN\%C3\%87\%C3\%830-DO-C\%C3\%82NCER-DE-COLO-DE-\%C3\%9ATERO-A-ATUA\%C3\%87\%C3\%830DO-PROFISSIONAL-ENFERMEIRO-NAS-UNIDADES-B\%C3\%81SICAS-DE-SA\%C3\%9ADE.pdf . Acesso em: 22 jan. 2020. 
BERTONCIM, K.R.I.; GASQUEZ, A.S.; LEITE, V.C. Indicadores de qualidade de exames colpocitológicos realizados por acadêmicos em uma clínica materno-infantil. Rev. UNINGÁ. .n. 56, v. 2, p. 172-183, 2019. Disponível em: http://revista.uninga.br/index.php/uninga/article/view/2161/1911. Acesso em: 10 dez. 2019.

BISPO, T.C.F.; OLIVEIRA, C.A.D.; NETO, E.A.F.; CARVALHO, E.R.; LINO, N.N.; SANTOS, S.M. Saúde sexual e reprodutiva no contexto da prisão. Rev. REC. Enf. Contemp. n. 4, v. 2, p. 109-120, 2016. Disponível em: https://www5.bahiana.edu.br/index.php/enfermagem/article/view/747/539. Acesso em: 22 jan. 2020.

BORGES, A.P.; ARENHARDT, K.; TERÇAS, A.C.P.; CABRAL, J.F.; LUCIETTO, G.C.; NASCIMENTO, V.F.; SILVA, R.A. Perfil socioeconômico e sexual de mulheres encarceradas. Rev. REME rev. Min. Enferm. n. 12, v. 7, p. 1978-1985, 2018. Disponível em: https://periodicos.ufpe.br/revistas/revistaenfermagem/article/ view/231408. Acesso em: 10 dez. 2019.

BRASIL. Ministério da Saúde. Instituto Nacional de Câncer José Alencar Gomes da Silva. Estimativa 2018: Incidência de câncer no Brasil. Coordenação e vigilância. Rio de janeiro, 2017. Disponível em: http://www.inca.gov.br/estimativa/2018/estimativa-2018.pdf. Acesso em: 13 jan. 2020.

BRASIL. Ministério da Saúde. Instituto Nacional de Câncer José Alencar Gomes da Silva. Diretrizes Brasileiras para o rastreamento do câncer do colo do útero. Coordenação de prevenção e vigilância: divisão de detecção precoce e apoio à organização de rede. Rio de janeiro, 2016. Disponível em: http:// www.citologiaclinica.org.br/site/pdf/documentos/diretrizes-para-o-rastreamento-do-cancer-do-colodo-utero_2016.pdf. Acesso em: 22 jan. 2020.

BRONSON, J.; CARSON, A. Prisioners in 2017. U.S. Office of Justice Programs, Bureau Justice Statistics, 2019. Disponível em: https://www.bjs.gov/. Acesso em: 10 dez. 2019.

CARVALHO, V.F.; KERBER, N.P.C.; WACHHLOZ, V.A.; POHLMANN, F.C.; MARQUES, L.A.; FRANCIONI, F.F. Acesso ao exame Papanicolau por usuários do Sistema Único de Saúde. Rev. Rene. n. 17, v. 2, p. 198-207, 2016. Disponível em: http://www.periodicos.ufc.br/rene/article/view/2999. Acesso em: 13 jan. 2020.

CERQUEIRA, J.C.; MOREIRA, J.P.L.; BRITO, A.S.; LUIZ, R.R. Indicador preventivo de saúde da mulher: proposta combinada de mamografia e Papanicolau. Rev. Panam. Salud. Pública. n. 41, p. 1-5, 2017. Disponível em: https://scielosp.org/pdf/rpsp/2017.v41/e99/pt. Acesso em: 13 jan. 2020.

CORRÊA, M.M.M.A.; FRANCISCO, J.C. Para além do encarceramento de idosas propostas humanizantes e educativas em presidio brasileiro. Rev. Inter. Ciênc. Humanas. n. 8, v. 1, p. 11-21, 2018. Disponível em: https://journals.epistemopolis.org/index.php/hu. Acesso em: 22 jan. 2020.

CORSINO, P.K.D.; NASCIMENTO, V.F.; LUCIETO, G.C.; HATTORI, T.Y.; GRAÇA, B.C.; ESPINOSA, M.M.; TERÇAS, A.C.P. Eficácia de ação educativa com reeducandas de cadeia pública de Mato Grosso sobre o vírus HPV. Rev. Saúde e pesqui. n. 11, p. 115-126, 2018. Disponível em: https://periodicos.unicesumar.edu. br/index.php/saudpesq/article/view/6372/3160. Acesso em: 13 jan. 2020.

EVARISTO, L.R.; DOMINGUES, E.A.R.; SILVA, R.S.; CARVALHO, M.R.F. Situações de saúde de mulheres privadas de liberdade em um município do sul de Minas Gerais. Rev. UninCor. n. 7, v. 2, 2017. Disponível em: http://periodicos.unincor.br/index.php/iniciacaocientifica/article/view/4850. Acesso em: 10 dez. 2019.

FRANCISCO, R.S. Homossexualidade feminina no cárcere: estratégias e (re) configurações de gênero como forma de sobrevivência. Rev. Vértices. n. 17, v. 1, p. 7-21, 2015. Disponível em: https://www. 
researchgate.net/publication/282208011_Female_homosexuality_the_case_of_the_Carlos_Tinoco_da_ Fonseca_female_prison_in_Campos_dos_Goytacazes_RJ. Acesso em: 13 jan. 2020.

GERMANO, I.M.P.; MONTEIRO, R.Á.F.G.; LIBERATO, M.T.C. Criminologia Crítica, feminismo e interseccionalidade na abordagem do aumento do encarceramento feminino. Rev. Psicol. Ciênc. Prof. n. 38, v. 2, p. 27-43, 2018. Disponível em: http://www.scielo.br/scielo.php?script=sci_arttext\&pid=S1414-98932018000600027. Acesso em: 22 jan. 2020.

GRAÇA BC, MARIANO MM, SILVA JH, NASCIMENTO VF, HATTORI TY, TERÇAS-TRETTEL ACP. PerfII epidemiológico e prisional das detentas de um município do médio norte de Mato Grosso. Rev. Semina Cienc. Biol. Saúde. n. 39, v.1, p. 9-68, 2018b.. Disponível em: http://www.uel.br/revistas/uel/index.php/ seminabio/article/view/30192. Acesso em: 13 jan. 2020.

GRAÇA, B.C.; MARIANO, M.M.; GUSMÃO, M.A.J.X.; CABRAL, J.F.; NASCIMENTO, V.F.; GLERIANO, J.S.; HATTORI, T.Y.; TERÇAS-TRETTEL, A.C.P. Dificuldades das mulheres privadas de liberdade no acesso aos serviços de saúde. Rev. Bras. Promoç. Saúde. n. 31, v. 2, p. 1-9, 2018a. Disponível em: http://periodicos. unifor.br/RBPS/article/view/7374. Acesso em: 22 jan. 2020.

GUIMARÃES, R.A.B.; SUCCI, G.M.; MONTALLI, V.A.M.; NIEDERAUER, A.J.S.; SUCCI, R.C.M. Resultados negativos na pesquisa cientifica: aspectos éticos. Rev. Bioét. n. 26, v. 2, p. 245-250, 2018. Disponível em: http://www. scielo.br/scielo.php?script=sci_arttext\&pid=S1983-80422018000200245. Acesso em: 13 jan. 2020.

INSTITUTE FOR CRIMINAL POLICY RESEARCH. Lista Mundial da população prisional. World Prison Brief, 2019. Disponível em: prisonstudies.org/news/icpr-launches-12th-edition-world-prison-population-list. Acesso em: 10 dez. 2019.

MAGALHÃES, M.C.; MAHL, G.H.; GEHM, L.L.; HERNANDES, C.P. Violência Sexual: vulnerabilidade e a conduta em serviço de saúde do Rio Grande do Sul. Rev. Prev. Infecç. Saúde. n. 3, v. 1, p. 23-28, 2017. Disponível em: http://www.ojs.ufpi.br/index.php/nupcis/article/view/5848./article/view/847/873. Acesso em: 22 jan. 2020.

OLIVEIRA, K.A.; SANTOS, L.R.C.S. Perfil epidemiológico da população carcerária feminina de vitória da conquista -BA. Rev. Saúde (Santa Maria). n. 42, v. 1, p. 21-30, 2016. Disponível em: https://periodicos. ufsm.br/revistasaude/article/view/15242. Acesso em: 13 jan. 2020.

ORGANIZAÇÃO MUNDIAL DA SAÚDE. Agencia Internacional de Pesquisa em Câncer. Câncer mundial relatório 2014: o global e regional fardo do câncer. Organização Mundial da Saúde, 2014. Disponível em: http//www.drugsandalcohol.ie/28525/1/World\%20Cancer\%20Report.pdf. Acesso em: 10 dez. 2019.

ORGANIZAÇÃO MUNDIAL DA SAÚDE. Câncer hoje: ficha de informação sobre a população: Brasil. Organização Mundial de Saúde, 2019b. Disponível em: http://gco.iarc.fr/today/data/factsheets/ populations/76-brazil-fact-sheets.pdf. Acesso em 22 jan. 2020.

ORGANIZAÇÃO MUNDIAL DA SAÚDE. Câncer hoje: folhas de fatos do câncer. cérvix uterina. Organização Mundial da Saúde, 2019a. Disponível em: http://gco.iarc.fr/today/data/factsheets/cancers/23-CervixUteri-fact-sheet.pdf. Acesso em: 10 dez. 2019.

PINTO, V.M.; GOLUB, J.E.; TANCREDI, M.V.; ALENCAR, R.S.; MIRANDA, A.E. Cevical Cytology and Histopathologic Abnormalities in Women Living AIDS in São Paulo, Brazil. J Acquir Immune Defic Syndr. n. 3, p. 212-216, 2011. Disponível em: https://www.ncbi.nlm.nih.gov/pubmed/21857321. Acesso em: 13 jan. 2020. 
ROSENDO, D.A.; LORENTE, S.; SANTO, C.M.; FERREIRA, G.M.; CANELLO, L.M.; ETLINGER-COLONELLI, D. Células escamosas atípicas de significados indeterminado (ASC-US): seguimento de exames analisados no instituto Adolfo Lutz. Rev. RBAC. n. 50, v. 3, p. 265-269, 2018. Disponível em: http://www.rbac.org. br/wp-content/uploads/2019/01/RBAC-vol-50-3-2018-ref-680.pdf. Acesso em: 10 dez. 2019.

SILVEIRA, B.L.; MAIA, R.C.B.; CARVALHO, M.F.A. Câncer do colo de útero: papel do enfermeiro na estratégia e saúde da família. Rev. FAEMA. n. 9, v. 1, p. 348-372, 2018. Disponível em: http://www.faema.edu.br/ revistas/index.php/Revista-FAEMA/article/view/517/484. Acesso em: 22 jan. 2020.

SILVEIRA, B.M.; MAIA, R.C.B.; CARVALHO, M.F.A. Câncer do colo de útero: papel do enfermeiro na estratégia e saúde da família. Rev. FAEMA. n. 9, v. 1, p. 348-72, 2018. Disponível em: http://www.faema.edu.br/ revistas/index.php/Revista-FAEMA/article/view/517/484. Acesso em: 13 jan. 2020.

SIMÕES, A.V.; SILVA, S.A.; TEIXEIRA, M.A. Prevenção do câncer cérvico-uterino: concepção de usuárias de unidade básica de saúde de um município baiano. Rev. Saude.com. v. 10 n. 4, p. 322-32, 2014. Disponível em: http://periodicos2.uesb.br/index.php/rsc/article/view/322/259. Acesso em: 22 jan. 2020.

TERÇAS, A.C.P.T.; NASCIMENTO, V.F.; GUSMÃO, M.A.J.X.; GRAÇA, B.C.; MARIANO, M.M.; GLERIANO, J.S. Na detenção ou na liberdade: onde eu encontro minha saúde. Investigación em Enfermería: Imagen y Desarollo. N. 21, v. 2, 2019. Disponível em: https://revistas.javeriana.edu.co/index.php/ imagenydesarrollo/article/view/19246. Acesso em: 10 dez. 2019. 